\title{
Theory of the elasticity of the materials of the second order
}

\author{
V. Shorkin ${ }^{1} \&$ V. Gordon ${ }^{2}$ \\ ${ }^{I}$ Department of Physics, Oryol technical states university, Russia \\ ${ }^{2}$ Department of Mathematics, Oryol technical states university, Russia
}

\begin{abstract}
It is admitted that a solid body is obtained by means of extraction from an infinite medium, and at the same time its state changes. To describe this phenomenon there a linear theory of elasticity of isotropic materials of the second order was used, generalized for the case of availability of the initial stressed state described by tensors of various ranks. They depend upon the physical nature of the body, the form of its boundary and position of points for their determination. The boundary effect - existence of surface tension and surface energy is described. The analysis of known test data regarding values of these quantities, wave dispersion in resilient media, calculus results on the basis of the model offered and one-dimensional theory of the crystalline lattice of elastic energy density for a number materials allowed values of elastic modulus and initial stresses to be determined. The created model of medium is used for the description of the stressed state of bodies adhered together, when their adhesion is considered to be one of the kinds of contact interaction. The calculus results of adhesion energy are confronted with the known data.

Keywords: theory of the elasticity, materials of the second order, the initial stressed state, and surface energy, adhesion energy.
\end{abstract}

\section{Introduction}

It is known that particles of solid body - atoms, ions, interact with the aid of the potential forces [1]. Therefore a change in the position at least of one particle of a body affects the position and the mutual influence of all remaining particles. The formation of the new section of free surface, for example, with the division, is accompanied by the separation of the sets of particles. On the one side, the 
adhesion of two bodies is connected with the association of large quantities of particles. In these processes the inter-atomic bonds break or are formed only along the interface or association. But the result affects all particles of the divided or united bodies. The possibility of the calculation of the aforesaid exists with the discrete description of the behavior of material [2]. This can be made, also, with the continuous non-local description $[3,4]$. But the first method is difficult to use for describing the materials with the complex structure. The second method requires information about the potential of non-local interaction. For describing the surface phenomena it is possible to use gradient theories (for example $[5,6])$. They use a higher order differential equation of motions. Surface effects can be considered as the effects of boundary layer. However, in the existing models is not traced clearly the connection of the predicted effects with the special features of potential interaction of the particles of solid body. In the model proposed below the underlining of this connection is the basis of theory.

\section{Model of continuous elastic medium}

\subsection{Basic assumptions}

1. Interaction of the atoms of real body is described with the aid of the potential of pair wise interaction. It is assumed that for the continuous simulator interaction of infinitely small particles is also potential, paired. The potential of interaction is proportional to their volumes $d V$ and $d V_{1}$ ( $\rho=$ const - material density), that have at the moment of the time $t$ of position $\vec{R}$ and $\vec{R}_{1}$. Constant of proportionality $\Phi=\Phi\left(\vec{R}_{1}, \vec{R}\right)$ (further potential) - the continuous and differentiated function of its arguments.

2. The studied processes are isothermal, reversed. The mechanical properties of medium are completely determined by the form of the function $\Phi=\Phi\left(\vec{R}_{1}, \vec{R}\right)$. The uniformity of properties indicates the dependence $\Phi\left(\vec{R}_{1}, \vec{R}\right)=\Phi\left(\vec{R}_{1}-\vec{R}\right), \vec{R}_{1}-\vec{R}=\vec{a}+\Delta \vec{u}$, where $\vec{a}=\vec{r}_{1}-\vec{r}-$ the state vector of particle $d V_{1}$ relatively $d V$ in the initial state of the considered body, $\Delta \vec{u}=\vec{u}_{1}-\vec{u}$ relative particle displacement upon transfer of body into the current state. Values $|\vec{u}|$ and $\left|\vec{u}_{1}\right|$ are considered small. Therefore $|\Delta \vec{u}|-$ also low value.

3. For the exception of the influence of boundary it is assumed that any body $B$ is obtained by isolation from infinitely extensive homogeneous continuous medium $\Omega$. Isolation occurs instantly. Potential $\Phi=\Phi\left(\vec{R}_{1}, \vec{R}\right)$ does not change. Initial for the body $B$ is the state, in which it was located after instantaneous isolation. The motion, caused by disruption of the equilibrium of internal forces, begins at this moment. It is considered that it rapidly goes out. Therefore, in further reasoning it is not examined. 


\subsection{Stress-strained state and mechanical properties}

The characteristics of the strained and stressed state of medium are introduced as follows. The potential $d \varphi$ of particle $d V$ inside the homogeneous medium is calculated through the formula

$$
d \varphi=\left[\int_{\Omega} \Phi\left(\vec{R}_{1}-\vec{R}\right) d V_{1}\right] d V
$$

Its variation relative to the current state of medium $\Omega$ is determined by the equality

$$
\begin{aligned}
\delta(d \varphi)=\delta w d V & =\left[\int_{\Omega}\left(\Phi\left(\vec{R}_{1}-\vec{R}+\delta(\Delta \vec{u})\right)-\Phi\left(\vec{R}_{1}-\vec{R}\right)\right) d V_{1}\right] d V= \\
& =\left[\int_{\Omega}\left(\nabla \Phi\left(\vec{R}_{1}-\vec{R}\right)\right)^{T} \cdot \delta(\Delta \vec{u}) d V_{1}\right] d V .
\end{aligned}
$$

Potential gradient $\vec{F}=\nabla \Phi\left(\vec{R}_{1}-\vec{R}\right)$ is written in the form

$$
\vec{F}\left(\vec{R}_{1}-\vec{R}\right)=\vec{F}(\vec{a})+\left.(\nabla \vec{F})^{T}\right|_{\Delta \vec{u}=0} \cdot \Delta \vec{u}
$$

In this case for the relative displacement $\Delta \vec{u}$ is considered valid the idea in the form Taylor series according to the external degrees of vector $\vec{a}$ [7]

$$
\Delta \vec{u}=\sum_{k=1}^{\infty} \frac{1}{k !}\left[\nabla_{\vec{r}}(k)_{\vec{u}}\right]_{\vec{a}=0} \cdot \ldots \cdot \vec{a}^{k}
$$

The consequence of these operations is the idea of a variation in the density of a change in the potential of internal forces $\delta w$, developing in the environment $d V$ of point $\vec{r}$, which is equal to the work of internal forces in this region, in the form

$$
\delta w=\sum_{m=1}^{\infty} P^{(m) T} \cdot \ldots \cdot \delta\left(\nabla_{\vec{r}}^{(m)} \vec{u}\right) .
$$

where: 


$$
\begin{gathered}
P^{(m)}=P^{0(m)}+\sum_{n=1}^{\infty} C^{(m, n) T} \cdot \ldots \cdot\left(\nabla^{\left.(n)_{\vec{r}} \vec{u}\right),}\right. \\
P^{0(m)}=\int_{\Omega}\left[\vec{F}(\vec{a}) \vec{a}^{m}\right] d V_{1}, \\
C^{(m, n)}=\frac{1}{n ! m !} \int_{\Omega} \vec{a}^{n}\left(\nabla_{\vec{a}} \vec{F}\right) \vec{a}^{m} d V_{1} .
\end{gathered}
$$

It follows from idea (5) that the infinite sequence $\left\{\nabla_{\vec{r}}(m)_{\vec{u}}\right\}$ of the gradients of displacements is the characteristic of the strained state in the infinitely small environment $d V$ of point $\vec{r}$. Its elements - these are the generalized displacements, determined in $d V$. By the generalized forces, which work on them, appear the stress tensors, determined by equalities (6) - (8). They compose the infinite sequence $\left\{P^{(m)}\right\}$ of the characteristics of the stressed state of region $d V$. It is characteristic that the calculation of interaction of the particles of the medium by the introduction of potential $\Phi=\Phi\left(\vec{R}_{1}, \vec{R}\right)$ led to the need for the calculation of the initial stressed state in the medium, caused exclusively by its intrinsic properties. These properties are described by function $\Phi=\Phi\left(\vec{R}_{1}, \vec{R}\right)$. It determines the values of the mechanical characteristics (in the classical understanding) of medium $\Omega$, computed with the aid of (8).

For the homogeneous infinite medium $\Omega$ tensors (7) and (8) do not depend on the position of the point of their determination. The same is correct for the body $B$, when isolated it in $\Omega$ mentally only with surface $S$ with the external unit normal $\vec{n}$. Picture changes with the real isolation, described in the third assumption of introduction. The action of the appearance of body $B$ on its particles disappears at this moment, integration in (7) and (8) must be produced not on $\Omega$, but on $B$. In this case the position of boundary $S$ relative to each point $\vec{r}$, from which is counted off the vector $\vec{a}$, proves to be different. In spite of the safety of function $\Phi=\Phi\left(\vec{R}_{1}, \vec{R}\right)$ with the real isolation of body $B$ from $\Omega$ it ceases to be uniform in the classical understanding.

\subsection{External actions and equation of motion}

The system of the differential equation of motions and boundary conditions takes the form

$$
\begin{array}{cc}
\nabla \cdot\left(P^{(1)}-\nabla \cdot\left(P^{(2)}-\nabla \cdot\left(P^{(3)}-\ldots\right)\right)\right)+\vec{f}=\rho \frac{\partial^{2} \vec{u}}{\partial t^{2}} & \vec{r} \in V, \\
\left.\vec{n} \cdot\left(P^{(1)}-\nabla \cdot\left(P^{(2)}-\ldots\right)\right)-\nabla_{S} \cdot\left(\vec{n} \cdot\left(P^{(2)}-\ldots\right)\right)\right)=\Pi^{(0)} & \vec{r} \in S, \\
\vec{n} \cdot\left(P^{(2)}-\nabla \cdot\left(P^{(3)}-\ldots\right)\right)-\nabla_{S} \cdot\left(\vec{n} \cdot\left(P^{(3)}-\ldots\right)\right)=\Pi^{(0)} & \vec{r} \in S .
\end{array}
$$


$\nabla \equiv \nabla_{\vec{r}}-$ the symbol of volumetric gradient; $\nabla_{S}-$ the symbol of surface gradient. It was assumed during the construction of system (9) - (11) that, as in the classical case, external actions on any body and any part of it are divided into those volumetrically distributed and those superficially distributed. The first make classical sense. Their bulk density is $\vec{f}$. The second, just as in the classical case, characterize contact interaction of different bodies. However, this interaction is characterized not by one form of forces, but by infinite sequence, that possess surface densities $\Pi^{(k)}, k=0,1, \ldots$. With $k=0$ this is the density of the classical surface forces, which accomplish work on the true displacements of material points. With $k>0$ - this is of density over forces [8]. System (9) - (11) must be augmented by the differential form of the law of conservation of momentum of pulse and by initial conditions for pour on displacements and their speeds.

\subsection{Version of the potential $\Phi=\Phi\left(\vec{R}_{1}, \vec{R}\right)$ determination}

It can be realized for the isotropic medium. In this case it suffices to examine one-dimensional situation. Equation (9) taking into account (6) - (8) which $\vec{f}=0$ takes the form

$$
\frac{1}{c^{2}} \frac{\partial^{2} u_{1}}{\partial t^{2}}=\frac{\partial^{2} u_{1}}{\partial x_{1}{ }^{2}}+b_{1}{ }^{2} \frac{\partial^{4} u_{1}}{\partial x_{1}{ }^{4}}+b_{2}{ }^{4} \frac{\partial^{6} u_{1}}{\partial x_{1}{ }^{6}}+\ldots
$$

Coefficients are determined by the formulas

$$
\begin{aligned}
& c=\sqrt{\frac{C_{11}^{(1,1)}}{\rho}}, \quad D_{j}=D_{1} b_{j-1}^{2(j-1)}, \quad j=1,2, \ldots, \\
& D_{1}=C_{11}^{(1,1)}, \quad D_{2}=C_{111}{ }^{(1,3)}-C_{111}{ }^{(2,2)}+C_{111}{ }^{(3,1)},
\end{aligned}
$$

The search for the solution in the form $u_{1}=e^{i(\omega t-k x 1)}, i=\sqrt{-1}$, gives the possibility to obtain dispersion law - dependence $\omega(k)[1]$, in the form of the power series

$$
\frac{\omega^{2}}{c^{2}}=k^{2}-b_{1}{ }^{2} k^{4}+b_{2}{ }^{4} k^{6}-\ldots
$$

The same law can be built theoretically, with the use of ideas of solid state physics [1], or on the basis of data of experiments [9]. With the comparison it is possible to determine values $b_{j}$. Function $\Phi=\Phi(a), a=|\vec{a}|$ can be 
represented, for example, in the form of a number of the diminishing exponential curves

$$
\Phi(a)=\sum_{p=1}^{\infty} \alpha_{p} e^{-\eta p}, \quad \eta_{p}=\frac{a}{b_{p}}
$$

Substitution (15) in (7), (8), and then in (13) reduces to the infinite system of linear algebraic equations for the unknowns $\alpha_{p}$. Its solution determines potential (15) of material, for which are obtained the values $b_{j}$.

\section{The theory application}

\subsection{Additional assumptions}

1. Instead of the infinite sequences of the generalized displacements and stresses they are used only on their two first elements. This gives the possibility to use for its solution classical methods, in particular, the method of separation of variables - Fourier's method. The analytical method of solving the ordinary differential equations of final order with the arbitrary differentiated coefficients, which are appeared in the one-dimensional case, is represented into [10].

2. Maximum simplification in the task is possible to achieve, if we assume that in the range of values in question under the condition of coefficients are constant. 2. It is assumed that the material is isotropic. In its near-boundary layer the values of the tensors of initial stresses and characteristics of elastic state are constant and equal to those values, which are characteristic for the boundary plane of semi-infinite body. In this case the density of a change in the internal forces potential is determined by the equality

$$
\begin{aligned}
w & =\mu g_{i j} g_{i j}+\frac{\lambda}{2} g_{k k} g_{l l}+ \\
& +(2 \mu+\lambda)\left(b_{1 \alpha}{ }^{2} Z_{i j k} Z_{i j k}+b_{1 \beta}{ }^{2} Z_{i j k} Z_{k i j}\right)+\pi_{0} \delta_{i j} \mathrm{E}_{k} Z_{i j k}
\end{aligned}
$$

$g_{i j}=\frac{1}{2}\left(u_{i, j}+u_{j, i}\right)$ and $Z_{i j k}=u_{i, j k}-$ the component of the classical strain tensor and second gradient of displacements; $\mu, \lambda-$ the Lame coefficients; $b_{1}, b_{2}, \pi_{0}$ - additional constants; $\mathrm{E}_{k}-$ the component of the unit vector, normal to the surface, parallel to boundary, where $\overrightarrow{\mathrm{E}}=\vec{n} ; P^{(1)} i j$ and $P^{(2)}{ }_{i j k}$ they are determined by differentiation (16) on $g_{i j}$ and $Z_{i j k}$ respectively. Relationship (16) corresponds to the model of the linearly elastic medium of the second order with the initial stressed state. 


\subsection{Quantity checking of theory}

Within the framework of the theory proposed is examined the task about the stress-strained state of semi-infinite body, on which act no external forces. On the basis of its solution and data of experiments regarding the surface energy $W_{p}$ of solid bodies [11] in accordance with the formulas

$$
\pi_{0}=\frac{W_{p}}{k}, b=\frac{3 W_{p}}{4 k^{2} A_{1}}, k=\lambda / 2 \mu+\lambda, 2 A_{1}=2 \mu+\lambda, b^{2}=b_{1 \alpha}{ }^{2}+b_{2 \beta}{ }^{2} .
$$

the estimations of additional elastic constants are made for the number of materials $b$ and $\pi_{0}$. The results of calculations are represented in table 1 .

Table 1: Estimations of additional elastic constants.

\begin{tabular}{|c|c|c|c|c|}
\hline № & Element & $W_{p}\left(D g / \mathrm{m}^{2}\right)$ & $b \cdot 10^{11}(\mathrm{~m})$ & $\pi_{0}\left(\mathrm{Dg} / \mathrm{m}^{2}\right)$ \\
\hline 1 & $\mathrm{Mg}$ & 0,73 & 2,67 & 1,28 \\
\hline 2 & $\mathrm{Al}$ & 1,04 & 4,65 & 1,92 \\
\hline 3 & $\mathrm{Si}$ & 1,24 & 7,54 & 3,21 \\
\hline 4 & $\mathrm{Ni}$ & 1,44 & 2,49 & 2,50 \\
\hline 5 & $\mathrm{Cu}$ & 1,12 & 1,89 & 1,57 \\
\hline 6 & $\mathrm{Ag}$ & 0,95 & 1,98 & 1,28 \\
\hline 7 & $\mathrm{Au}$ & 1,23 & 1,29 & 1,46 \\
\hline
\end{tabular}

The use of a dispersion law [1] for determining the parameter $b$ leads to the results, commensurate with those given.

\subsection{Adhesion of two solid bodies}

By adhesion of two solid deformed bodies $B^{(1)}$ and $B^{(2)}$ in the model of medium is understood this form of their contact, when the rectilinear material fiber, normal to the surface of contact $S_{a}$ and which intersects it, in the process of the deformation of the united of body $B=B^{(1)} \cup B^{(2)}$ preserves not only its integrity (as in the case of classical, solid contact), but also smoothness of the distribution of its deformations. This idea gives the possibility to solve the problem about the stress-strained state of the body, which are in a state of adhesion as the combined task about the state of piecewise-uniform body $B=B^{(1)} \cup B^{(2)}$ with the condition of the absence of external actions on it. Adhesion energy $F$ of bodies $B^{(1)}$ and $B^{(2)}$ is determined by loss per the unit surface area, along which occurred the adhesion, their free energy [12]

$$
F=W_{p}^{(1)}+W_{p}^{(2)}-W_{p}^{(1,2)} .
$$


Here: $W_{p}^{(1,2)}-$ the surface energy of the system of bodies, concentrated bi $S_{a}$; $W_{p}^{(j)}$ - the surface energies of the disconnected bodies. The theory, examined in this work, makes it possible to consider the volumetric distribution of these is specific internal energy by means of calculated value (16). For two plates with the thickness, for much the great significance of the parameters $b^{(j)}$, is obtained the formula

$$
|F|=\frac{W_{p}^{(1)} W_{p}^{(2)}\left(k^{(1)}+k^{(2)}\right)^{2}}{W_{p}^{(1)} k^{(2) 2}+W_{p}^{(2)} k^{(1) 2}}, k^{(j)}=\frac{v^{(j)}}{1-v^{(j)}},
$$

$v^{(j)}$ - Poisson ratio. The results of the comparison of the results of theory with the data of experiment are represented in table 2 .

Table 2: The comparison of the results of theory with the data of experiment.

\begin{tabular}{|c|c|c|}
\hline Metal & $\begin{array}{c}\text { adhesion energy on silicon } \\
\left(\mathrm{Dg} / \mathrm{m}^{2}\right)\end{array}$ & $\begin{array}{c}\text { adhesion energy on the } \\
\text { glass }\left(\mathrm{Dg} / \mathrm{m}^{2}\right)\end{array}$ \\
\hline aluminum $(\mathrm{Al})$ & 0,214 & 0,150 \\
\hline silver $(\mathrm{Ag})$ & 0,181 & 0,120 \\
\hline copper $(\mathrm{Cu})$ & 0,210 & 0,110 \\
\hline
\end{tabular}

\section{Conclusion}

At present is discussed a question about the correspondence of the discrete and continuous description of real material [2]. In the represented work it is shown that it is possible to attain an increase in the degree of the adequacy of description by the allotment of continuous medium with the properties, characteristic for the discrete medium. In this work this property is the ability of particles to interact at the significant distance with the aid of the potential forces. The gradient model of the second order for the medium with the initial stresses became the result this of calculation. It proved to be applicable for the use in the calculations of surface energy and adhesion energy.

\section{References}

[1] Kittel, Ch., Introduction to Solid State Physics, Science: Moscow, pp 111-148, 1978.

[2] Krivtsov, A.M. \& Myasnikov, V.P., Simulation by the method of the dynamics of particles of the change in the internal structure and stressed 
state in the material under the strong thermal influence. By notify of Russia academy of science. Mechanic of solid body, 1, pp. 88 - 103, 2005.

[3] Gordon, V.A. \& Shorkin, V.S., The non-local theory of the surface layer of solid body. Proceedings of Ttua State University. Mathematic. Mechanic. Informatics. 4(2), pp. 55 - 57, 1998.

[4] Eringen, A. C., Nonlocal continuum field theories, Springer-Verlag: Berlin, 2002.

[5] Mikaelyan, K.N., Gutkin, M. Yu \& Ayfantis, Ye. S., Edge dislocations at the phase boundaries in the gradient theory of elasticity. Solid state physics, 2(9), pp. 1613 - 1620, 2000.

[6] Vitcovsky, I.V, Konev, A.N., Shorkin, V.S., Kzaev, N.D., Rusanov, A.E., Khoroshikh, V.M., \& Leonov. S.L. Adhesion energy estimation of some composite materials. Plasma Devices and Operations, 11(2), pp 81-87. 2003.

[7] Korn, G. \& Korn, T., Mathematical handbook for scientists and engineers, eMGRAW-HILL BOOK COMPANY, INC: New York, Toronto, London, 1961.

[8] Tupin, R.A., The theory of elasticity, which considers moment stresses. Voltages the mechanics coll. of transfers, 3, pp. 113 - 140, 1965.

[9] Woods, A.D.B., Cochran, W. \& Brockhouse, B.N. Lattice Dynamics of Alkali Halide Crystals. Phys. Rev., 119, pp.980-999, 1960.

[10] Gordon, V.A., Shorkin V.S. \& Borsenkov, M.I., The method of solution of problems of mechanics of heterogeneous bodies, Oryol State University: Oryol, pp. $35-46,2005$.

[11] Samsonov, G.V., (ed). Properties of elements. Physical Property, Metallurgy: Moskow, 1976.

[12] Vvedensky, B.A., (ed). Physical encyclopedias dictionary, Soviet encyclopedia: Moskow, 1960. 